\title{
Organic electronic synapses with pinched hystereses based on graphene quantum-dot nanocomposites
}

\author{
Hwan Young Choi, Chaoxing Wu, Chang Han Bok and Tae Whan Kim \\ Organic electronic synapses (e-synapses) based on poly(3,4-ethylenedioxythiophene) polystyrene sulfonate (PEDOT:PSS)/ \\ graphene quantum dot (GQD) nanocomposites are fabricated by using a solution method. Current-voltage ( $I-\eta$ curves for the \\ devices under dual positive bias voltage sweeps show that the conductance with a pinched hysteresis gradually increased with \\ increasing applied voltage, and those for the devices under dual negative bias voltage sweeps gradually decreased with \\ increasing applied voltage, indicative of the fingerprint of e-synapses. The current in the devices decreases with increasing \\ concentration of GQDs in the active layer, and the devices fabricated utilizing the ratio of PEDOT:PSS to GQDs of 1:0.4 shows \\ the best performance among the e-synapses. The carrier transport and operating mechanisms of the e-synapses are described on \\ the basis of both the $I-V$ results and the trapping and escape of electrons from the GQDs.
}

NPG Asia Materials (2017) 9, e413; doi:10.1038/am.2017.133; published online 28 July 2017

\section{INTRODUCTION}

The fabrication of bio-inspired, cognitive, adaptive solid-state devices, which form the basis for synaptic electronics, a field of research aiming to build artificial synaptic devices to emulate the neuromorphic computation utilizing biological synapses, has been investigated extensively. ${ }^{1-4}$ Synapses dominate the structure of the brain, and they are responsible for the massive parallelism, structural plasticity and robustness of the brain. Furthermore, the synapses play a crucial role in performing the biological computations that underlie perception and learning. Various kinds of device systems with programmable conductance inspired by existing device technologies, such as phasechange memories, resistive-change memories, ferroelectric switches, carbon-nanotube devices, and three-terminal devices or field-effecttransistor-based devices, have been explored. Among those types of programmable memory devices, resistive switching devices, due to their simple structures, are currently the best candidates for realizing the function of the synapses. ${ }^{5,6}$

Resistive switching devices provide potential capabilities both to change the Si-based computing industry in the fields of logic and analog nonvolatile memories and to play an important role in biologically inspired structures, including electronic synapses and neuromorphic integrated circuits. ${ }^{7-10}$ Resistive switching materials significantly affect the electrical characteristics of the devices. Among the various resistive switching materials, carbon-based nanocomposites have appeared as promising candidates for applications in bioinspired electronics because of their low cost, environmental friendliness, mechanical flexibility, ductility and compatibility with complementary metal-oxide semiconductor technology. ${ }^{11-13}$ Furthermore, nanoscale-size carbon materials, including graphene, graphene oxide, graphene quantum dots (GQDs) and carbon nanotubes, have attracted attention because their electronic doping properties, energy levels and charge-trapping processes can be precisely engineered by changing the size of the particles. ${ }^{14}$ In particular, GQDs show superior properties of excitation-dependent photoluminescence, ${ }^{15}$ variations in energy levels, electroluminescent properties ${ }^{16}$ and charge-storage capabilities for potential applications in electronics and optoelectronics. ${ }^{17,18}$ The charge-storage capability of GQDs, which originates from the presence of charge-trapping states, ${ }^{19-22}$ affects the transport properties of polymer nanocomposites embedded with GQDs. Even though a few studies on the electrical characteristics of organic electronic synapses (e-synapses) have been performed, ${ }^{23}$ very few investigations concerning the operating mechanisms of organic e-synapses based on poly (3,4-ethylenedioxythiophene) polystyrene sulfonate (PEDOT:PSS)/ GQD nanocomposites have to be performed. This paper reports data for the pinched hystereses and the operating mechanisms of organic e-synapses based on PEDOT:PSS/GQD nanocomposites as an active layer. $I-V$ measurements were performed to investigate the fingerprint of the e-synapses. The effects of the concentration of GQDs on the electrical characteristics of the e-synapses were observed. The carrier transport and operating mechanisms of the e-synapses were described on the basis of the $I-V$ results and the carrier transport processes in the energy band diagram.

\section{MATERIALS AND METHODS}

A mixed solution containing a PEDOT:PSS solution (Clevios ph 1000; Heraeus, Hanau, Germany) and a GQD suspension in deionized water (ACS Material, Pasadena, CA, USA) was prepared to fabricate a PEDOT:PSS/GQD hybrid layer. Various ratios of PEDOT:PSS to GQDs in the mixed solution were prepared using $1 \mathrm{ml}$ of PEDOT:PSS and 0, 0.2, 0.4, 0.6, 1 and $1.2 \mathrm{ml}$ of GQDs to investigate the effect of GQD concentration on the performance of the 

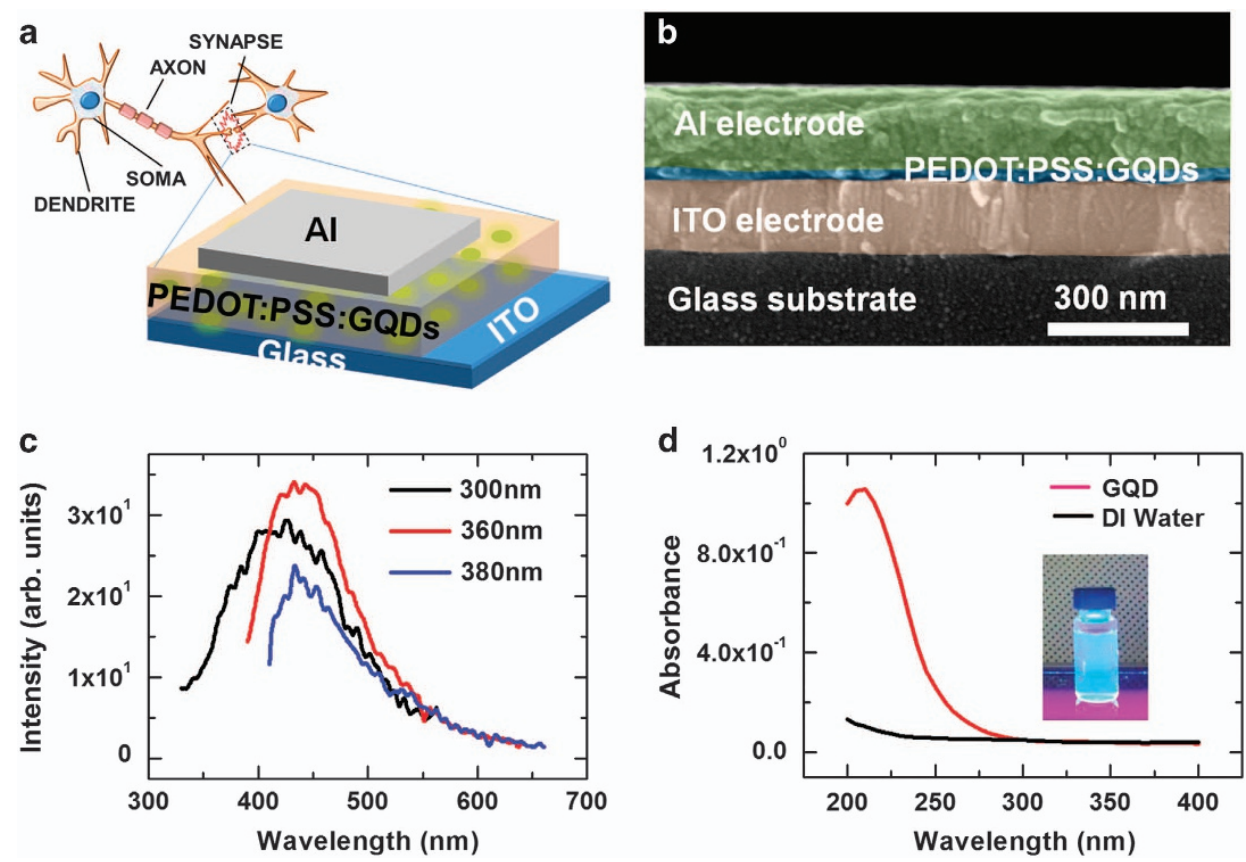

Figure 1 (a) Structure of neuron and schematic diagram of the Al/PEDOT:PSS:GQDs/ITO/glass device. (b) Cross-sectional scanning electron microscopy image of the device. (c) Photoluminescence spectra of the GQDs. (d) UV-vis spectra of the GQDs and the deionized water. The inset presents the GQDs under 365-nm UV illumination exhibited bright blue emission.
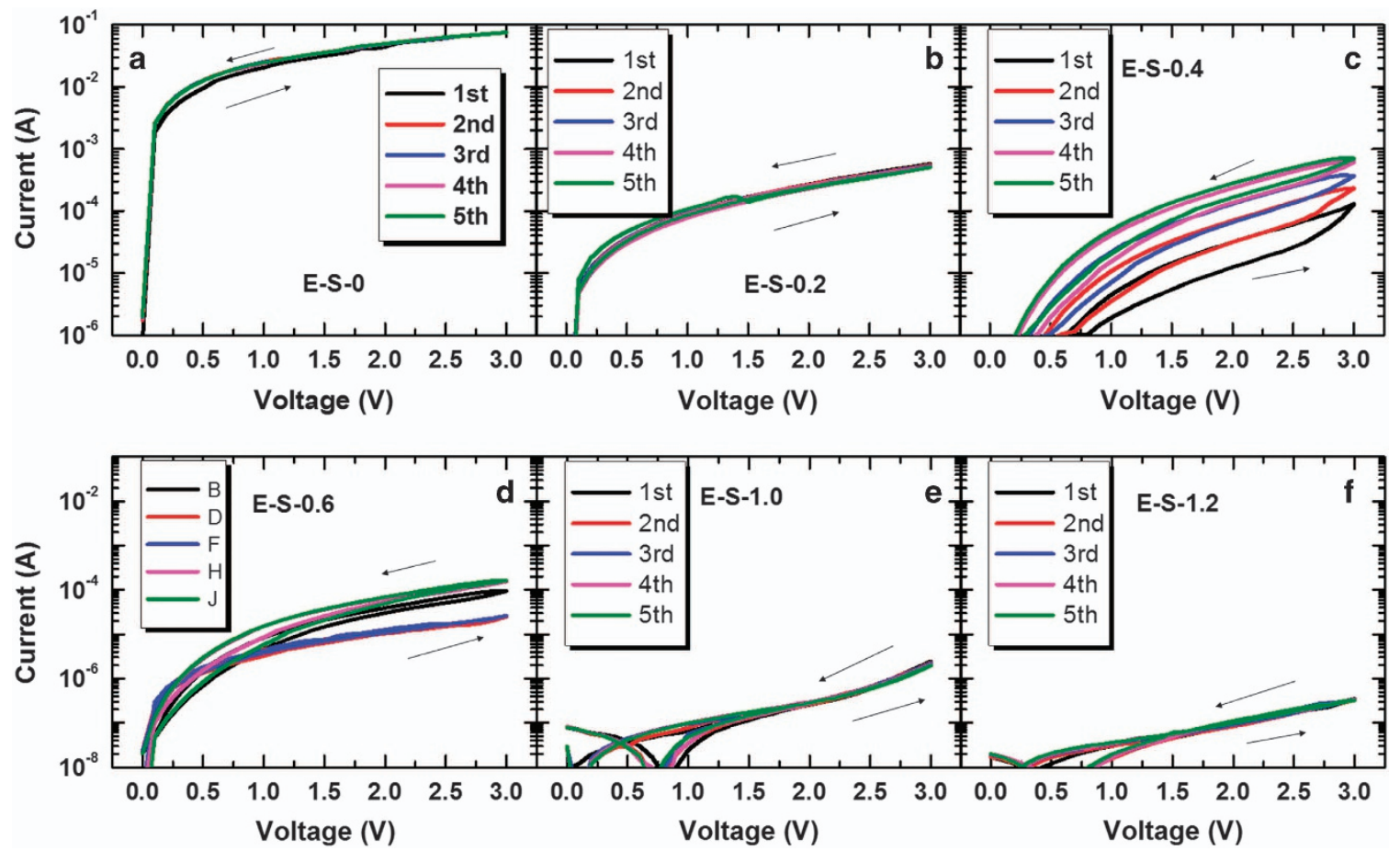

Figure 2 Current-voltage $(I-V)$ curves for the devices with different GQD concentrations. The ratios of PEDOT:PSS to GQDs are 1 to (a) 0 , (b) 0.2 , (c) 0.4 , (d) 0.6 , (e) 1 and (f) 1.2 .

e-synapses. The devices utilizing diverse mixed solutions with GQD concentrations of $0,0.2,0.4,0.6,1$ and $1.2 \mathrm{ml}$ are denoted as E-S-0, E-S-0.2, E-S-0.4, E-S-0.6, E-S-1.0 and E-S-1.2, respectively. The mixed solution was ultrasonicated for $30 \mathrm{~min}^{24}$ Indium-tin-oxide (ITO) substrates were sonicated in acetone, methanol and deionized water in sequence for $30 \mathrm{~min}$ for the fabrication of the e-synapses. The cleaned ITO substrates were dried with blowing $\mathrm{N}_{2}$ gas. The PEDOT:PSS/GQD active layers were deposited on the ITO substrates by using a spin-coating method at 3000 r.p.m. for $30 \mathrm{~s}$ and then at 300 r.p.m. for 10 s. After spin coating, the solvent was removed by heating the devices on a hot plate at $100^{\circ} \mathrm{C}$ for $10 \mathrm{~min}$. Finally, Al electrodes with a diameter of $1 \mathrm{~mm}$ and a thickness of $200 \mathrm{~nm}$ were deposited on the active layer by using a thermal evaporation method. The electrical properties were investigated at room temperature in the atmosphere by using a Keithely 2400 system. Scanning electron microscopy images were observed by using a Nova 
a

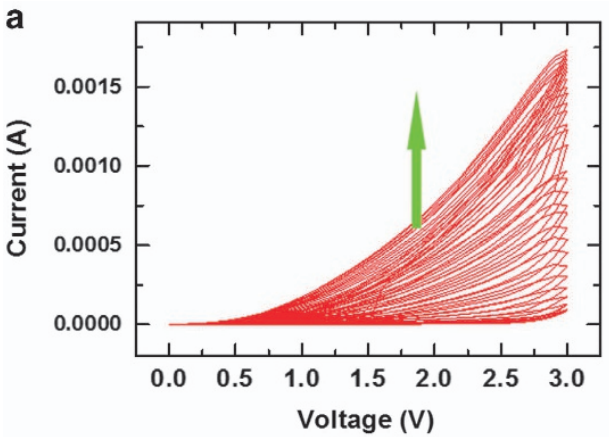

C

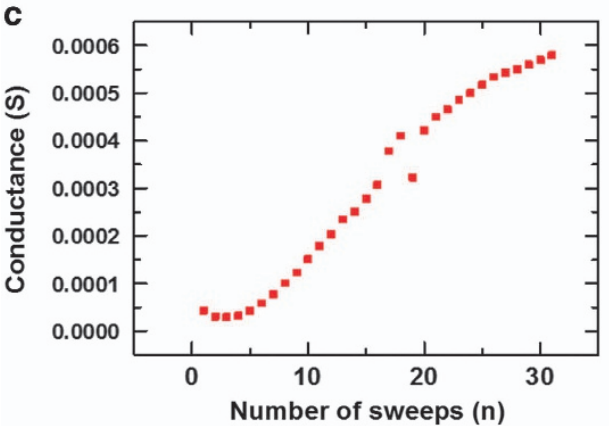

b
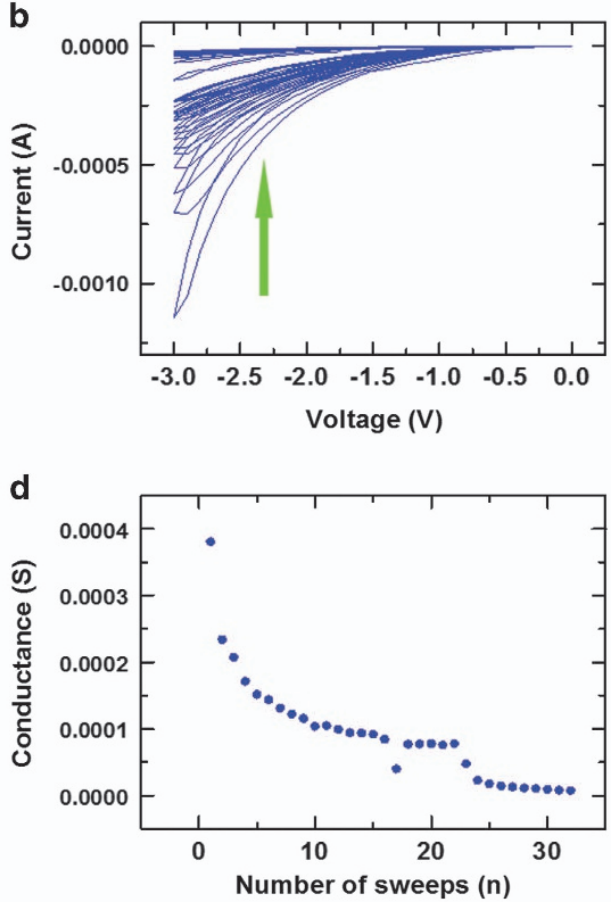

Figure 3 Current-voltage $(I-V$ curves under positive and negative dual sweeps. (a) $I-V$ curves under positive dual sweep. (b) $I-V$ curves under a negative voltage dual sweep. Conductance as a function of the number of sweeps for the devices under (c) positive dual sweeps and (d) negative dual sweeps.

Nano SEM 450 system (FEI, Hillsboro, OR, USA). Ultraviolet photoelectron spectroscopy spectra were measured by using an X-ray photoelectron spectroscopy-theta probe, and ultraviolet-visible (UV-vis) absorption spectra were investigated by using a Lambda 650S (Perkin Elmer, Waltham, MA, USA).

\section{RESULTS AND DISCUSSION}

Figure 1a is a structure of neuron and the schematic structure of the device fabricated in this study. The top $\mathrm{Al}$ electrodes were grounded for the $I-V$ measurements, and the bias voltage was applied to the bottom ITO electrode. The thickness of the active layer in the device was approximately $43 \mathrm{~nm}$, as shown in the scanning electron microscopy image of Figure 1b. The PEDOT:PSS/GQD active layer was uniformly deposited on the ITO bottom electrode. Al electrodes of $200 \mathrm{~nm}$ thickness and $1 \mathrm{~mm}$ diameter were deposited on the active layer. Figure 1c presents the photoluminescence spectra of the GQDs. Figure 1d presents the UV-vis spectra of the GQDs and the deionized water. The inset presents the GQDs under $365 \mathrm{~nm}$ UV illumination exhibited bright blue emission. The UV-vis spectrum of the GQD is quite different from that of the deionized water. Supplementary Figure S1 in the Supplementary Information shows that the GQDs are smaller than $10 \mathrm{~nm}$ in size.

The concentration of the GQDs embedded in a conducting polymer can significantly affect the electrical characteristics. Figures $2 \mathrm{a}-\mathrm{f}$ present the $I-V$ curves of the devices for various GQD concentrations. Dual voltage sweeps of $0-3-0 \mathrm{~V}$ were applied to the devices in each measurement. The E-S-0 device showed large current values, as shown in Figure 2a, because PEDOT:PSS is a highly conducting polymer. ${ }^{25,26}$ The current in the devices decreased with increasing concentration of GQDs. The current in the E-S- 0.2 device decreased to about $0.5 \mathrm{~mA}$ under $3 \mathrm{~V}$, as shown in Figure $2 \mathrm{~b}$, and the initial current for the E-S-0.4 device decreased further, as shown in Figure 2c. For the E-S-0.4 device, the initial current for the first curve (the black one) reached $6 \times 10^{-7} \mathrm{~A}$ at $3 \mathrm{~V}$, and its maximum current increased with increasing number of dual voltage sweeps. The maximum currents at
$3 \mathrm{~V}$ of the first, second, third, fourth and fifth $I-V$ curves for that device were $1.3 \times 10^{-4}, 2.3 \times 10^{-4}, 3.7 \times 10^{-4}, 6.1 \times 10^{-4}$ and $7.03 \times 10^{-4} \mathrm{~A}$, respectively. The gradual variation in the conductance with increasing number of applied voltage sweeps is a natural feature of e-synapses. ${ }^{27,28}$ The $I-V$ curves for the E-S-0.6 device show that the behavior of the e-synapse still exhibited a slightly imperfect pinched hysteresis, as shown in Figure $2 \mathrm{~d}$. The current of the e-synapse under $3 \mathrm{~V}$ continuously decreased to approximately $1 \times 10^{-5} \mathrm{~A}$ with increasing GQD concentration. The current of the E-S-1.0 device under an applied voltage of $3 \mathrm{~V}$ was about $2.2 \times 10^{-6} \mathrm{~A}$, as shown in Figure 2e, and the pinched hysteresis for the E-S-1.0 device disappeared. The current of the E-S-1.2 device further decreased to $3.4 \times 10^{-7} \mathrm{~A}$ under $3 \mathrm{~V}$, as shown in Figure $2 \mathrm{f}$. The current of the devices gradually decreased by approximately tenfold with increasing GQD concentration in the PEDOT:PSS, as shown in Figures 2a-f, indicative of the GQDs acting as trap sites, resulting in an increased resistance in the active layer. The $I-V$ curves for the E-S- 0.4 device show the best shape of the pinched hysteresis curve, indicative of the characteristic behavior of e-synapses.

The detailed performance of the e-synapse for the optimized device is presented in Figure 3. Figures $3 \mathrm{a}$ and $\mathrm{b}$ show the $I-V$ curves of the E-S-0.4 devices under positive and negative dual voltage sweeps. Supplementary Figures S3a and b show Figures $3 \mathrm{a}$ and b, respectively, on a $\log (I) \sim V$ scale. Figure $3 c$ shows the conductance as a function of the number of applied positive voltage dual sweeps and Supplementary Figure S3c also presents the log value of conductance with number of positive voltage dual sweeps. Figure $3 \mathrm{~d}$ shows the conductance as a function of the number of applied negative voltage dual sweeps and Supplementary Figure S3d also presents the log value of conductance with number of negative voltage dual sweeps. Figure $3 c$ and Supplementary Figure S3c represent the currents in the E-S-0.4 device under an applied voltage of $3 \mathrm{~V}$, and Figure $3 \mathrm{~d}$ and Supplementary Figure S3d show those under an applied voltage of 

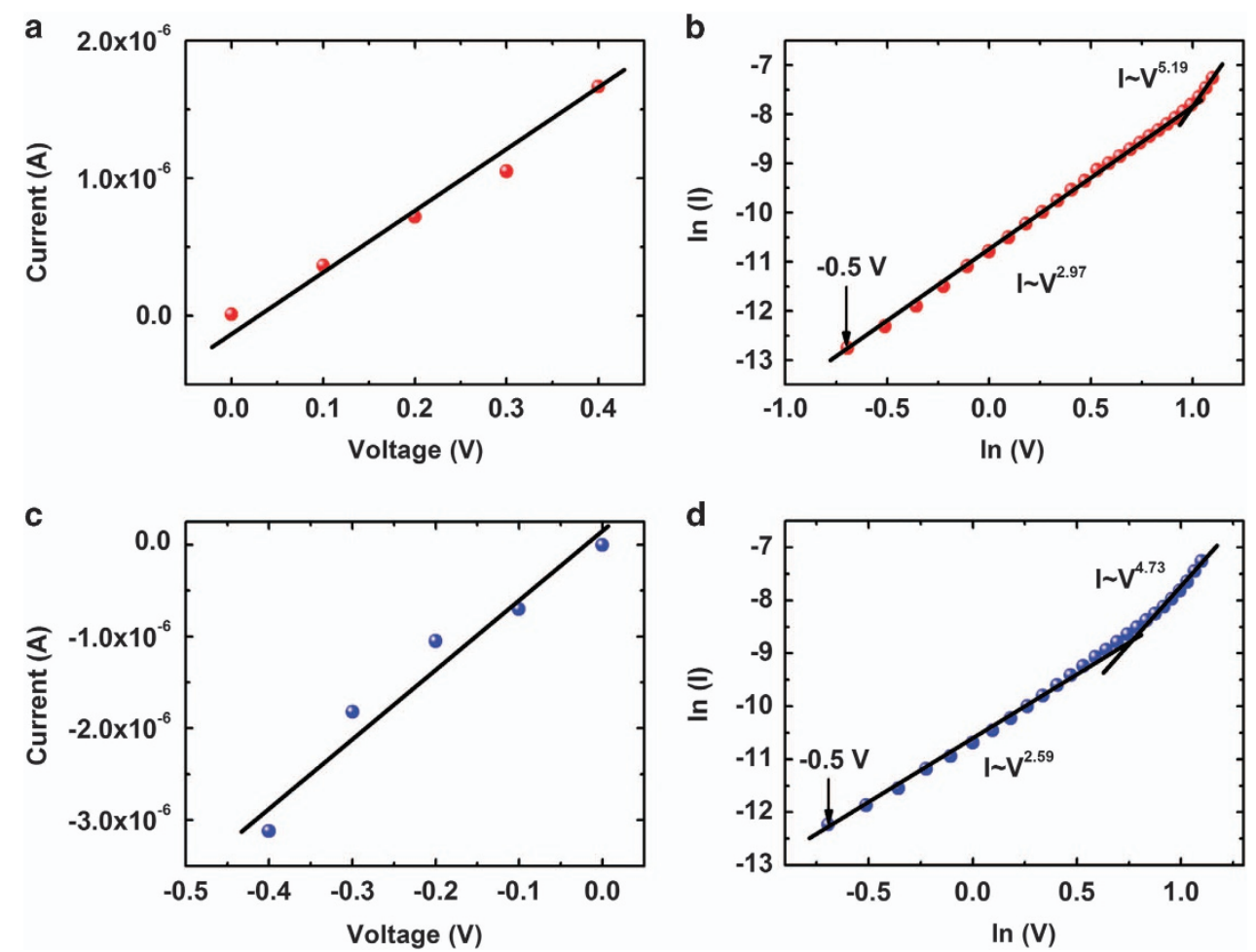

Figure 4 Current-voltage fittings of the (a) $L-V$ curve for the devices under a positive bias voltage below $0.5 \mathrm{~V}$, the (b) In $(I) \sim \ln (V)$ curve under a positive bias voltage from 0.5 to $3 \mathrm{~V}$, the (c) $I-V$ curve under a negative bias voltage below $-0.5 \mathrm{~V}$ and the (d) In $(I) \sim \ln (V$ curve under a negative bias voltage from -0.5 to $-3 \mathrm{~V}$.
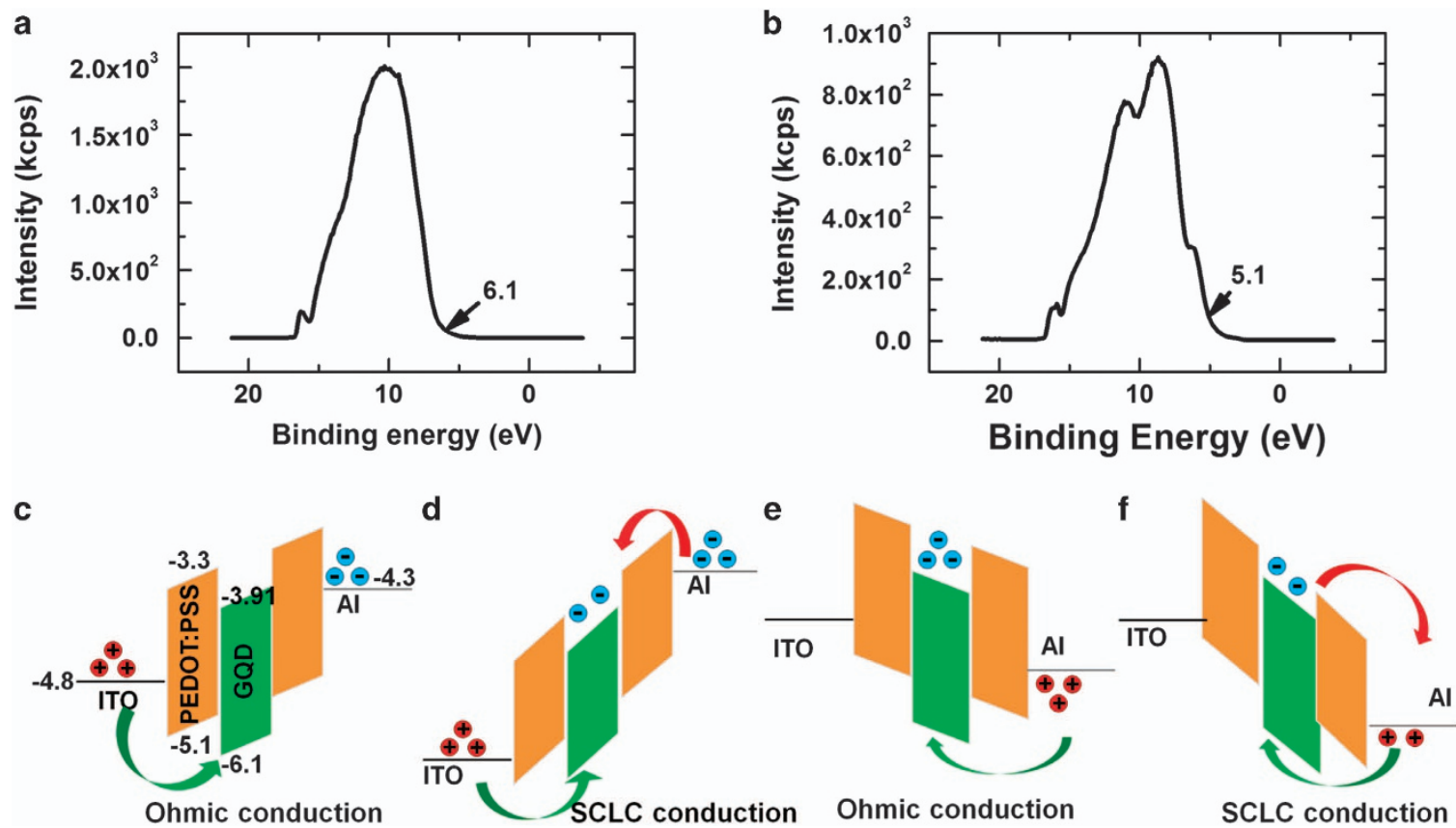

Figure 5 Ultraviolet photoelectron spectroscopy spectra of (a) the GQDs and (b) the PEDOT:PSS. Schematic diagrams of the carrier transport behaviors for the devices (c) under small positive bias voltages, (d) under larger positive bias voltages, (e) under small negative bias voltages and (f) under larger negative bias voltages.

$-3 \mathrm{~V}$. Firstly, when a positive $0-3-0 \mathrm{~V}$ dual sweep sequence is applied, the current increases with increasing number of voltage dual sweeps, as shown in Figure 3a. All of the $I-V$ curves for the E-S-0.4 device show counterclockwise hysteresis behaviors, indicating that a high- resistance state for the forward sweep in the positive bias range changes to a low-resistance state (LRS) for the applied reverse sweep. Therefore, the resistance state of the device gradually decreases with increasing number of voltage dual sweep, as shown in Figures $3 \mathrm{a}$ 
and c. The resistance of the LRS is almost the same as that of the high-resistance state under the previous positive dual voltage sweep. When a positive dual-sweep voltage is applied to the device, the variation in the resistance state for the device follows the same trend. Thus, the electrical characteristics are related to the typical electrical properties of memristive devices used as synapses.

The clockwise hystereses of the $I-V$ curves for the E-S-0.4 device under a negative voltage $0-3-0 \mathrm{~V}$ dual sweep sequence are presented in Figure 3b, and the resistance can be seen to simultaneously increase. The LRS of the $I-V$ curves for the device at the initial stage changed to a high-resistance state after $-3 \mathrm{~V}$, which is opposite to the results for positive dual voltage sweeps. In addition, the resistance of the high-resistance state is almost the same as that of the LRS under the previous negative dual voltage sweep. This phenomenon means that the variation in the resistance state of the device under applied negative dual voltage sweeps can be remembered, which can also be attributed to a characteristic memristive behavior similar to that under positive dual voltage sweeps, as shown in Figures $3 \mathrm{~b}$ and $\mathrm{d}$. The $I-V$ curves of the device under both forward and reverse dual voltage sweeps are nonlinear, which is a useful feature to mitigate the sneak path currents in a large crossbar array. As a result, the conductance of the device under both positive and negative dual voltage sweeps increases or decreases. The incremental increase or decrease of the conductance for the device is analogous to the potentiation or depression of the synapses in neuromorphic systems. ${ }^{27-29}$

$I-V$ fittings were performed in order to clarify the carrier transport mechanisms of the e-synapses. Figures $4 \mathrm{a}-\mathrm{d}$ are graphs that were fitted in order to obtain the $\alpha$-value of $I \sim V^{\alpha}$ to investigate the carrier transport mechanisms in the devices. Figures $4 \mathrm{a}$ and $\mathrm{b}$ present the fitted graphs under a positive dual voltage sweep. Firstly, the $I-V$ curve is linear below $0.5 \mathrm{~V}$, as shown in Figure $4 \mathrm{a}$, indicating that Ohmic conduction dominates the carrier transport in the device at the initial state of the forward sweep. Because the $\alpha$-value of the device above an applied voltage of $0.5 \mathrm{~V}$ is 2.97 , the conduction is dominated by the space-charged-limited-current (SCLC) mechanism. ${ }^{30}$ The $\alpha$-value increases to 5.19 with increasing applied voltage, indicating that more space charges are formed in the active layer. The $I-V$ curve of the device under a negative dual voltage sweep is linear below $-0.5 \mathrm{~V}$; thus, below $-0.5 \mathrm{~V}$, the device at the initial state is characterized by Ohmic conduction, just as it was under a positive dual voltage sweep. Because the $\alpha$-value of the fitted curves changes from 2.59 to 4.73 with increasing voltage above $-0.5 \mathrm{~V}$, the device follows the SCLC mechanism, as shown in Figure $4 \mathrm{~d}$.

The possible operating mechanisms of the e-synapses can be described on the basis of the above results. Figures $5 \mathrm{a}$ and $\mathrm{b}$ show the ultraviolet photoelectron spectroscopy spectra of the GQD and the PEDOT:PSS, respectively. The band-gap energy of the GQD is obtained from the UV-vis spectrum, and the highest occupied molecular orbital levels of the GQD and the PEDOT:PSS are determined from the ultraviolet photoelectron spectroscopy spectra. ${ }^{31}$ The energy level of the lowest unoccupied molecular orbital of the GQD is determined by using the experimental results for its band-gap energy and the energy level of its highest occupied molecular orbital is in reasonable agreement with the value reported in ref. 32. The energy level of the lowest unoccupied molecular orbital of the PEDOT:PSS is determined from the experimental highest occupied molecular orbital level of the PEDOT:PSS and the energy gap taken from ref. 33 as shown in Figure $5 c$. Figures $5 c-f$ show the energy band diagrams of the device to explain the carrier conduction mechanisms. Only holes in an initial state with an insufficient positive voltage bias can be injected from the ITO into the highest occupied molecular orbital of the PEDOT:PSS. As a result, the device undergoes Ohmic conduction, as shown in Figure 5c. Electrons are also injected from the $\mathrm{Al}$ electrodes to the PEDOT:PSS with increasing applied voltage, subsequently becoming trapped in the GQDs. As a result, the SCLC mechanism is dominant in the device, as shown in Figure $5 \mathrm{~d}$. The trap-controlled SCLC causes a dramatic increase in the conductivity of the nanocomposites, resulting in the setting of the system in the LRS, as can be seen in Figure $3 \mathrm{a}^{34}$ More electrons are trapped in the GQDs with increasing positive dual voltage sweep, which leads to a gradual increase in the working current. The device under a negative dual voltage sweep conducts as an Ohmic conductor with increasing voltage until $-0.5 \mathrm{~V}$, as shown in Figure $5 \mathrm{e}$, similar to the conduction mechanism of the device under a positive dual voltage sweep. The SCLC mechanism dominates the conduction for a strong reverse sweep, as shown in Figure 5f. However, the electrons trapped in the GQDs under a negative dual voltage sweep are released, resulting in a decrease in the working current.

\section{CONCLUSION}

The electrical characteristics of the e-synapse with a Al/PEDOT:PSS: GQD/ITO/glass structure were investigated. The current of the e-synapse decreased with increasing GQD concentration because the GQDs acted as trap sites. The $I-V$ curves of the devices showed that the pinched hysteresis loops of the $I-V$ curves crossed the origin and that the conductance with a pinched hysteresis increased with increasing applied voltage, a fingerprint of a memristor. The carrier transport mechanism of the e-synapses was dominated by SCLC conduction resulting from electron trapping by the GQDs. The operating mechanisms of the e-synapses could be described on the basis of both energy band diagrams and the trapping and escape of electrons from the GQDs. The electrical properties of the devices can help improve understanding of the promising results for potential applications in next-generation electrical synapses.

\section{CONFLICT OF INTEREST}

The authors declare no conflict of interest.

\section{ACKNOWLEDGEMENTS}

This research was supported by Basic Science Research Program through the National Research Foundation of Korea (NRF) funded by the Ministry of Education, Science and Technology (2016R1A2A1A05005502).

\section{PUBLISHER'S NOTE}

Springer Nature remains neutral with regard to jurisdictional claims in published maps and institutional affiliations.

1 Widrow, B. \& Hoff, M. E. Adaptive switching circuits. IRE Western Electron. Show Convention, Convention Record Part 4, 96-104 (1960).

2 Steinbuch, K. \& Peter, M. US Patent No. 3,286,238 (1966).

3 Stanford, R. \& Ovshinsky, I. M. Analog models for information storage and transmission in physiological systems. Mater. Res. Bull. 5, 681-690 (1970).

4 Wang, Z., Joshi, S., Savel'ev, S. E., Jiang, H., Midya, R., Lin, P., Hu, M., Ge, N., Strachan, J. P., Li, Z., Wu, Q., Barnell, M., Li, G. L., Xin, H. L., Williams, R. S., Xia, Q. \& Yang, J. J. Memristors with diffusive dynamics as synaptic emulators for neuromorphic computing. Nat. Mater. 16, 101-108 (2017).

5 Borghetti, J., Li, Z., Straznicky, J., Li, X., Ohlberg, D. A., Wu, W., Stewart, D. R. \& Williams, R. S. A hybrid nanomemristor/transistor logic circuit capable of self-programming. Proc. Natl Acad. Sci. USA 106, 1699-1703 (2009).

6 Kim, T. W., Yang, Y., Li, F. \& Kwan, W. L. Electrical memory devices based on inorganic/organic nanocomposites. NPG Asia Mater. 4, e18 (2012).

7 Chua, L. Memristor-the missing circuit element. IEEE Trans. Circuits Syst. 18, 507-519 (1971). 
8 Chen, Y., Liu, G., Wang, C., Zhang, W., Li, R. W. \& Wang, L. Polymer memristor for information storage and neuromorphic applications. Mater. Horiz 1, 489-506 (2014).

9 Jo, S. H., Chang, T., Ebong, I., Bhadviya, B. B., Mazumder, P. \& Lu, W. Nanoscale memristor device as synapse in neuromorphic systems. Nano Lett. 10, 1297-1301 (2010).

10 Choi, B. J., Zhang, J., Norris, K., Gibson, G., Kim, K. M., Jackson, W., Zhang, M.-X. M., Li, Z., Yang, J. J. \& Williams, R. S. Trilayer tunnel selectors for memristor memory cells. Adv. Mater. 28, 356-362 (2016).

$11 \mathrm{Wu}, \mathrm{C} ., \mathrm{Li}, \mathrm{F}$. \& Guo, T. Efficient tristable resistive memory based on single layer graphene/insulating polymer multi-stacking layer. Appl. Phys. Lett. 104, 183105 (2014).

12 Wu, C., Li, F., Guo, T. \& Kim, T. W. Controlling memory effects of three-layer structured hybrid bistable devices based on graphene sheets sandwiched between two laminated polymer layers. Org. Electron. 13, 178-183 (2012).

13 Wu, C., Li, F., Zhang, Y., Guo, T. \& Chen, T. Highly reproducible memory effect of organic multilevel resistive-switch device utilizing graphene oxide sheets/polyimide hybrid nanocomposite. Appl. Phys. Lett. 99, 042108 (2011).

14 Eustis, S. \& El-Sayed, M. A. Why gold nanoparticles are more precious than pretty gold: noble metal surface plasmon resonance and its enhancement of the radiative and nonradiative properties of nanocrystals of different shapes. Chem. Soc. Rev. 35 209-217 (2006)

15 Bacon, M., Bradley, S. J. \& Nann, T. Graphene quantum dots. Part. Part. Syst. Charact. 31, 415-428 (2014).

16 Zhang, X., Zhang, Y., Wang, Y., Kalytchuk, S., Kershaw, S. V., Wang, Y., Wang, P. Zhang, T., Zhao, Y., Zhang, H., Cui, T., Wang, Y., Zhao, J., Yu, W. W. \& Rogach, A. L. Color-switchable electroluminescence of carbon dot light-emitting diodes. ACS Nano 7 , 11234-11241 (2013).

17 Kim, J. K., Kim, S. J., Park, M. J., Bae, S., Cho, S. P., Du, Q. G., Wang, D. H., Park, J. H. \& Hong, B. H. Surface-engineered graphene quantum dots incorporated into polymer layers for high performance organic photovoltaics. Sci. Rep. 5, 14276 (2015)

18 Gupta, V., Chaudhary, N., Srivastava, R., Sharma, G. D., Bhardwaj, R. \& Chand, S. Luminscent graphene quantum dots for organic photovoltaic devices. J. Am. Chem. Soc. 133, 9960-9963 (2011).

19 Kim, Y. R., Jo, Y. E., Shin, Y. S., Kang, W. T., Sung, Y. H., Won, U. Y., Lee, Y. H. \& Yu, W. J. Electrostatically transparent graphene quantum-dot trap layers for efficient nonvolatile memory. Appl. Phys. Lett. 106, 103105 (2015).

20 Kalita, H., Harikrishnan, V., Shinde, D. B., Pillai, V. K. \& Aslam, M. Hysteresis and charge trapping in graphene quantum dots. Appl. Phys. Lett. 102, 143104 (2013).

21 Joo, S. S., Kim, J., Kang, S. S., Kim, S., Choi, S. H. \& Hwang, S. W. Graphene-quantum-dot nonvolatile charge-trap flash memories. Nanotechnology 25, 255203 (2014).

22 Ji, Y., Kim, J., Cha, A. N., Lee, S. A., Lee, M. W. Suh, J. S., Bae, S. K. \& Wang, G. Graphene quantum dots as a highly efficient solution-processed charge trapping medium for organic nano-floating gate memory. Nanotechnology 27, 145204 (2016).

23 La Barbera, S., Vuillaume, D. \& Alibart, F. Filamentary switching: synaptic plasticity through device volatility. ACS Nano 9, 941-949 (2015).
24 Kepić, D. P., Marković, Z. M., Jovanović, S. P., Peruško, D. B., Budimir, M. D., Holclajtner-Antunović, I. D., Pavlović, V. B. \& Marković, B. M. T. Preparation of PEDOT: PSS thin films doped with graphene and graphene quantum dots. Synth. Met. 198, 150-154 (2014).

25 Williams, R. S. How we found the missing memristor. IEEE. Spectrosc. 45, 28-35 (2008).

26 Kim, Y. H., Sachse, C., Machala, M. L., May, C., Müller-Meskamp, L. \& Leo, K. Highly conductive PEDOT: PSS electrode with optimized solvent and thermal post-treatment for ITO-free organic solar cells. Adv. Funct. Mater. 21, 1076-1108 (2011).

27 Chang, T., Jo, S. H., Kim, K. H., Sheridan, P., Gaba, S. \& Lu, W. Synaptic behaviors and modeling of a metal oxide memristive device. Appl. Phys. A. Mater. Sci. Process. 102, 857-863 (2011).

28 Chang, T., Jo, S. H. \& Lu, W. Short-term memory to long-term memory transition in a nanoscale memristor. ACS Nano 5, 7669-7676 (2011).

29 Biolek, Z. \& Biolek, D. How can the hysteresis loop of the ideal memristor be pinched? IEEE Trans. Circuits. Syst. II. Express Briefs 61, 491-495 (2014).

30 Rose, A. Space-charge-limited currents in solids. Phys. Rev 97, 1538 (1955).

31 Tadayyon, S. M., Grandin, H. M., Griffiths, K., Coatsworth, L. L., Norton, P. R., Aziz, H. \& Popovic, Z. D. Reliable and reproducible determination of work function and ionization potentials of layers and surfaces relevant to organic light emitting diodes. Org. Electron. 5, 199-205 (2004).

32 Sekiya, R., Uemura, Y., Naito, H., Naka, K. \& Haino, T. Chemical functionalisation and photoluminescence of graphene quantum dots. Chemistry 22, 8198-8206 (2016).

33 Ratcliff, E. L., Meyer, J., Steirer, K. X., Armstrong, N. R., Olson, D. \& Kahn, A. Energy level alignment in PCDTBT: PC 70 BM solar cells: solution processed NiOx for improved hole collection and efficiency. Org. Electron. 13, 744-749 (2012).

34 Obreja, A. C., Cristea, D., Mihalache, I., Radoi, A., Gavrila, R., Comanescu, F. \& Kusko, C. Charge transport and memristive properties of graphene quantum dots embedded in poly (3-hexylthiophene) matrix. Appl. Phys. Lett. 105, 083303 (2014).

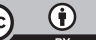

This work is licensed under a Creative Commons Attribution 4.0 International License. The images or other third party material in this article are included in the article's Creative Commons license, unless indicated otherwise in the credit line; if the material is not included under the Creative Commons license, users will need to obtain permission from the license holder to reproduce the material. To view a copy of this license, visit http:// creativecommons.org/licenses/by/4.0/

(C) The Author(s) 2017

Supplementary Information accompanies the paper on the NPG Asia Materials website (http://www.nature.com/am) 